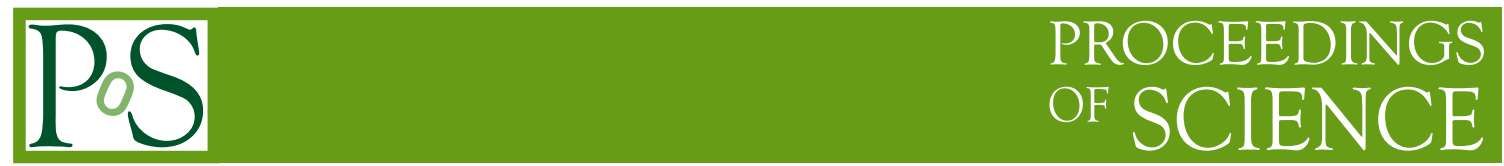

\title{
$\mathscr{N}=1$ supersymmetric Yang-Mills theory on the lattice
}

\section{Georg Bergner}

University of Frankfurt, Institute for Theoretical Physics, Max-von-Laue-Str. 1, D-60438 Frankfurt am Main, Germany

\section{Istvan Montvay}

Deutsches Elektronen-Synchrotron DESY,

Notkestr. 85, D-22603 Hamburg, Germany

\section{Gernot Münster*, Dirk Sandbrink, Umut D. Özugurel}

University of Münster, Institute for Theoretical Physics,

Wilhelm-Klemm-Str. 9, D-48149 Münster, Germany

E-mail: munsteg@uni-muenster.de

\begin{abstract}
Numerical simulations of supersymmetric theories on the lattice are intricate and challenging with respect to their theoretical foundations and algorithmic realisation. Nevertheless, the simulations of a four-dimensional supersymmetric gauge theory have made considerable improvements over the recent years. In this contribution we summarise the results of our collaboration concerning the mass spectrum of this theory. The investigation of systematic errors allows now a more precise estimate concerning the expected formation of supersymmetric multiplets of the lightest particles. These multiplets contain flavour singlet mesons, glueballs, and an additional fermionic state.
\end{abstract}

31st International Symposium on Lattice Field Theory LATTICE 2013

July 29 - August 3, 2013

Mainz, Germany

*Speaker. 


\section{Introduction}

Supersymmetry (SUSY) is a central concept in models for physics beyond the Standard Model of elementary particle physics. Supersymmetric theories also provide us with intriguing theoretical structures. Supersymmetry is an extension of the Poincaré symmetry of space-time corresponding to an extension of the Poincaré algebra by one or several supersymmetry charges to a superPoincaré algebra that relates bosons to fermions. The supercharges change the spin by $\frac{1}{2}$, hence the supersymmetry multiplets contain particles with different spins, in particular bosons and fermions, at the same time. Since at present energies no such supermultiplets with degenerate masses are observed, supersymmetry - if it is realised in Nature - has to be a broken symmetry.

Part of supersymmetric extensions of the Standard Model is the $\mathscr{N}=1$ supersymmetric YangMills theory (SYM). It is the supersymmetric extension of Yang-Mills theory. In addition to the gluons it contains their superpartners, the gluinos. Gluinos are Majorana fermions in the adjoint representation of the gauge group. The Lagrangian of SYM in the continuum is

$$
\mathscr{L}=\operatorname{tr}\left[-\frac{1}{4} F_{\mu v} F^{\mu v}+\frac{\mathrm{i}}{2} \bar{\lambda} \gamma^{\mu} D_{\mu} \lambda-\frac{m_{g}}{2} \bar{\lambda} \lambda\right]
$$

where $F_{\mu \nu}$ is the non-Abelian field strength formed out of the gauge fields $A_{\mu}(x), \lambda(x)$ is the gluino field, and $D_{\mu}$ denotes the gauge covariant derivative in the adjoint representation. The gluino mass term breaks the supersymmetry of the theory softly. For vanishing gluino mass it is expected that supersymmetry is not broken in SYM in the continuum [1].

$\mathscr{N}=1 \mathrm{SYM}$ is similar to QCD in various respects [2], the difference being the Majorana nature of the fermions and their colour representation. As in QCD the gauge coupling in SYM is asymptotically free at high energies and becomes strong in the infrared limit. Due to confinement the spectrum of particles is expected to consist of colourless bound states. If supersymmetry is unbroken the particles should belong to mass degenerate SUSY multiplets.

Many predictions concerning the properties of SYM are based on perturbation theory or semiclassical methods. The low-energy properties, in particular the structure of the particle multiplets are, however, of a non-perturbative nature. It would therefore be desirable to study whether the theory exists as a continuum limit of a non-perturbatively defined model regularised on a space-time lattice, and whether the predictions about its physical properties can be confirmed. In particular, to verify the formation of supermultiplets is a central task for non-perturbative studies in the lattice regularisation. Other non-perturbative properties of interest include the spontaneous breaking of chiral symmetry, $Z_{2 N_{c}} \rightarrow Z_{2}$, that manifests itself in the non-vanishing vacuum expectation value $\langle\lambda \lambda\rangle \neq 0$, the confinement of static quarks, indicated by a linear rise in the static quark potential, which is an evidence for the confining nature of the theory, and the validity of the supersymmetric Ward identities.

Results on these questions have been obtained by our collaboration in previous studies in the framework of a lattice-regularised version of SYM. Presently we study SYM with gauge group $\mathrm{SU}(2)$. Our non-perturbative studies are concentrating on the properties of the light particle spectrum. In particular, we determine the masses of the lightest composite particles by performing numerical simulations. For our recent publications see Refs. [3, 4, 5, 6, 7]. 


\section{SYM on the lattice}

The lattice discretisation in general breaks supersymmetry [8]. Models that respect part of an extended superalgebra are discussed in Ref. [9]. For $\mathscr{N}=1$ SYM supersymmetry is broken in any known lattice formulation. Our investigations are based on the Curci-Veneziano lattice action [10], which is built in analogy to the Wilson action of QCD [11] for the gauge field ("gluon") and Wilson fermion action for the gluino. It is given by

$$
S=S_{g}+S_{f}
$$

Here $S_{g}$ is the gauge field action

$$
S_{g}=\beta \sum_{p l}\left(1-\frac{1}{N_{c}} \operatorname{Retr} U_{p l}\right)
$$

with the gauge coupling $\beta \equiv 2 N_{c} / g^{2}$ for an $\mathrm{SU}\left(N_{c}\right)$ gauge field. $U_{p l}$ is the product of the gauge link fields along a plaquette. In our simulations we actually use the tree-level Symanzik improved gauge action, which contains, besides the plaquettes, also rectangular Wilson loops of perimeter six. The fermionic part of the action (2.1) is

$$
\begin{aligned}
S_{f} & \equiv \frac{1}{2} \bar{\lambda} Q \lambda \\
& \equiv \frac{1}{2} \sum_{x}\left\{\bar{\lambda}_{x}^{a} \lambda_{x}^{a}-K \sum_{\mu=1}^{4}\left[\bar{\lambda}_{x+\hat{\mu}}^{a} V_{a b, x \mu}\left(1+\gamma_{\mu}\right) \lambda_{x}^{b}+\bar{\lambda}_{x}^{a} V_{a b, x \mu}^{T}\left(1-\gamma_{\mu}\right) \lambda_{x+\hat{\mu}}^{b}\right]\right\} .
\end{aligned}
$$

Here $K$ is the hopping parameter which determines the gluino mass, $\gamma_{\mu}$ denotes a Dirac matrix and $V_{x \mu}$ is the gauge field variable in the adjoint representation of the gauge group, which is obtained from the gauge field links in the fundamental representation $U_{x \mu}$ by

$$
V_{x \mu}^{a b} \equiv 2 \operatorname{tr}\left(U_{x \mu}^{\dagger} T^{a} U_{x \mu} T^{b}\right)
$$

( $T^{a}$ are the generators of $\mathrm{SU}\left(N_{c}\right)$ ). The gluino field $\lambda_{x}$ satisfies the Majorana condition

$$
\bar{\lambda}_{x}=\lambda_{x}^{T} C
$$

with the charge conjugation Dirac matrix $C$. In the simulations we apply one or three levels of stout smearing to the link fields in the Wilson-Dirac operator.

Performing the path integral over the fermion field $\lambda$ results in a Pfaffian:

$$
\int[d \lambda] \mathrm{e}^{-\frac{1}{2} \bar{\lambda} Q \lambda}=\int[d \lambda] \mathrm{e}^{-\frac{1}{2} \lambda M \lambda}=\operatorname{Pf}(M),
$$

where $M$ is the antisymmetric matrix defined as

$$
M \equiv C Q=-M^{T}
$$

The square of the $\operatorname{Pfaffian} \operatorname{Pf}(M)$ is equal to the determinant of the fermion matrix $Q$ :

$$
\operatorname{det}(Q)=\operatorname{det}(M)=[\operatorname{Pf}(M)]^{2} .
$$


The lattice discretisation breaks both supersymmetry and chiral symmetry, but they are expected to be restored in the continuum limit if the hopping parameter is tuned to a critical value $K_{c}$ corresponding to a vanishing gluino mass. The breaking of chiral symmetry and the related need to tune the hopping parameter could be avoided by using domain-wall [12, 13] or overlap [14] fermions, but the SUSY breaking remains and the required numerical effort for simulations would substantially increase.

In the continuum the Pfaffian introduced above is positive, but on the lattice this is not necessarily the case. Therefore in the numerical simulations its sign has to be taken into account separately. Taking the non-negative square root of the determinant, the effective gauge field action is [10]:

$$
S_{C V}=\beta \sum_{p l}\left(1-\frac{1}{N_{c}} \operatorname{Retr} U_{p l}\right)-\frac{1}{2} \log \operatorname{det} Q[U] .
$$

The factor $\frac{1}{2}$ in front of $\log \operatorname{det} Q$ corresponds to a flavour number $N_{f}=\frac{1}{2}$ of Dirac fermions. The gauge configuration for this fractional flavour number can be created, for instance, by the two-step polynomial Hybrid Monte Carlo (TSPHMC) algorithm [15], which is our choice for Monte Carlo updating. The omitted sign of the Pfaffian can be taken into account by reweighting:

$$
\langle A\rangle=\frac{\langle A \operatorname{signPf}(M)\rangle_{C V}}{\langle\operatorname{signPf}(M)\rangle_{C V}}
$$

where $\langle\ldots\rangle_{C V}$ denotes expectation values with respect to the effective gauge action $S_{C V}$. This reweighting could in principle lead to a sign problem if a strong cancellation occurs among contributions with opposite sign. In previous work $[16,17]$ we have shown by monitoring the sign of the Pfaffian that for positive gluino masses the positive contributions dominate and there is practically no sign problem.

\section{Light particle spectrum}

The main interest of our present investigations of SYM is in the low-lying spectrum of particles. Colour neutral bound states can be created from the vacuum by gauge invariant operators which are built from the gluon and gluino field operators. These include the adjoint mesons a- $f_{0}$ and $a-\eta^{\prime}$. They are composed of two gluinos with spin-parity $0^{+}$and $0^{-}$, respectively. The corresponding bilinear operators are $\bar{\lambda} \lambda$ and $\bar{\lambda} \gamma_{5} \lambda$. The meson propagator has connected and disconnected contributions:

$$
\begin{aligned}
C_{\Gamma}(t) & =\frac{1}{V_{s}} \sum_{\vec{x}, \vec{y}}\langle\underbrace{\operatorname{tr}_{s c}\left[\Gamma Q_{x x}^{-1}\right] \operatorname{tr}_{s c}\left[\Gamma Q_{y y}^{-1}\right]}_{\text {disconnected }}-2 \underbrace{\operatorname{tr}_{s c}\left[\Gamma Q_{x y}^{-1} \Gamma Q_{y x}^{-1}\right]}_{\text {connected }}\rangle \\
& -\frac{1}{V_{s}}\left\langle\frac{1}{T} \sum_{t} \sum_{\vec{x}} \operatorname{tr}_{s c}\left[\Gamma Q_{x x}^{-1}\right]\right\rangle^{2},
\end{aligned}
$$

where $\operatorname{tr}_{s c}$ denotes a trace over spin and colour indices, and $\Gamma=1$ or $\gamma_{5}$, respectively. The numerical evaluation of the disconnected propagators is rather demanding. In order to reduce the large variance, the disconnected part has been calculated using the stochastic estimator method [18] 
combined with a truncated eigenmode approximation. As it is the case in $\mathrm{QCD}$, the disconnected diagrams are intrinsically noisier than the connected ones and dominate the level of noise in the total correlator.

Other particles which are expected to exist in this model are the glueballs, which are created by purely gluonic operators. In particular, one expects low-lying $0^{+}$and $0^{-}$glueballs. In addition to scalar and pseudoscalar particles, a chiral supermultiplet would contain a Majorana fermion particle. Such particles are provided by the gluino-glueballs. They are represented by the operator

$$
\tilde{O}_{g \tilde{g}}=\sum_{\mu v} \sigma_{\mu v} \operatorname{tr}\left[F^{\mu v} \lambda\right]
$$

where $\sigma_{\mu v}=\frac{1}{2}\left[\gamma_{\mu}, \gamma_{v}\right]$ and $F^{\mu v}$ is the field strength tensor. A lattice version of this, which can be used in numerical simulations, is

$$
O_{g \tilde{g}}^{\alpha}=\sum_{i<j, \beta} \sigma_{i j}^{\alpha \beta} \operatorname{tr}\left[P_{i j} \lambda^{\beta}\right]
$$

where the indices $i$ and $j$ stand for the spatial directions. A choice for $F_{i j}$ with the proper parity and time reversal transformation properties is the antihermitian part of the clover plaquette $U^{(c)}$

$$
P_{i j}=\frac{1}{8 \mathrm{i} g_{0}}\left(U_{\mu \nu}^{(c)}-\left(U_{\mu \nu}^{(c)}\right)^{\dagger}\right) .
$$

For its definition and more details see Ref. [5].

On the basis of effective Lagrangeans, in Refs. $[19,20]$ it has been predicted that the lowlying particles form two chiral supermultiplets, each consisting of a scalar, a pseudoscalar, and a fermionic spin $1 / 2$ particle. One of them contains an a $-\eta^{\prime}$, an a- $f_{0}$, and a gluino-glueball, the other one a $0^{-}$glueball, a $0^{+}$glueball, and a gluino-glueball.

In our numerical investigations we have calculated the masses of the gluino-glueball, a- $\eta^{\prime}$, $\mathrm{a}-f_{0}$, and $0^{+}$glueball. In addition the mass of the adjoint pion $(\mathrm{a}-\pi)$ is obtained. The correlator of this particle is given by the connected contribution of the $a-\eta^{\prime}$ correlator. The $a-\pi$ is not a physical particle in SYM. However, on the basis of arguments involving the OZI-approximation of SYM [19], the adjoint pion mass is expected to vanish for a massless gluino and the behaviour $m_{\mathrm{a}-\pi}^{2} \propto m_{g}$ can be assumed for light gluinos. The corresponding value of $K_{c}$ is most easily obtained from the dependence of the a- $\pi$-mass on $K$.

The gluino-glue correlator has been obtained using different smearing techniques. The link fields are smeared using APE smearing, the fermionic fields using Jacobi smearing. In order to decrease lattice artefacts and statistical fluctuations in the Wilson-Dirac fermion matrix $Q$ of the lattice action, the gauge link variables $U_{x \mu}$ have been replaced by stout smeared links [21].

In Ref. [5] a detailed investigation of finite size effects was done, showing that the lattice volumes used in our simulations are sufficiently large, such that the finite volume effects are smaller than the statistical errors.

The simulations are performed at non-zero gluino masses where supersymmetry is softly broken. The obtained masses are then extrapolated to vanishing gluino mass at $K=K_{c}$. The following figures show the masses of the particles discussed above as a function of the squared mass of the adjoint pion, which is a convenient substitute for the gluino mass. The inverse bare gauge coupling 

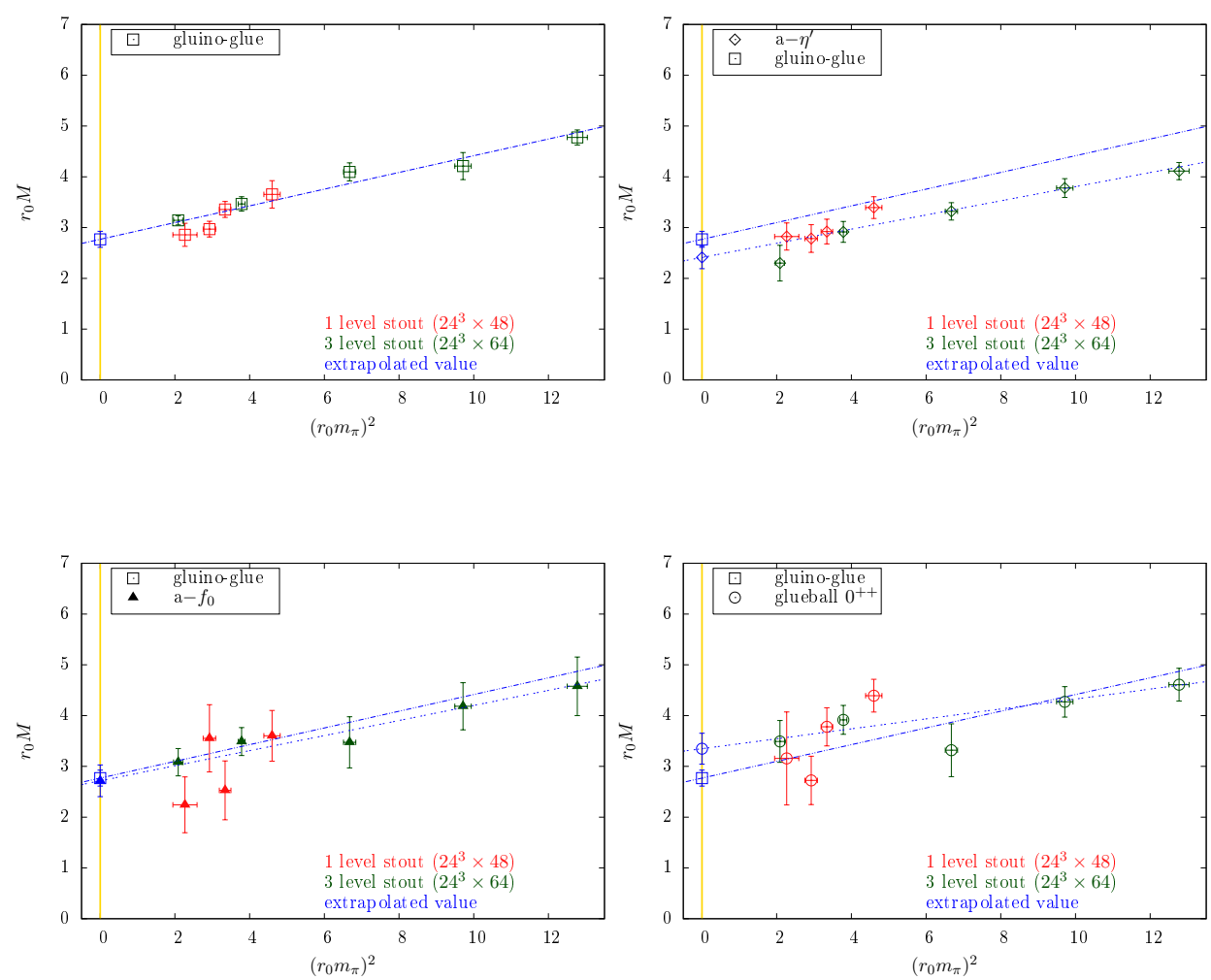

Figure 1: Light particle masses in SYM as a function of the squared mass of the adjoint pion. $r_{0}$ is the Sommer scale parameter.

in the current simulations was $\beta=1.75$. The scale is set by the Sommer parameter $r_{0}$, obtained from the static quark potential. We use QCD units by setting the Sommer parameter to $r_{0}=0.5 \mathrm{fm}$. The lattice spacing is approximately $0.055 \mathrm{fm}$ for 1 level, and approximately $0.058 \mathrm{fm}$ for 3 levels stout smearing. Also shown in the figures are the extrapolations to the limit of vanishing gluino mass. All figures include the gluino-glue mass for comparison.

\begin{tabular}{c|cccc}
$\beta$ & $\mathrm{a}-\eta^{\prime}$ & $\mathrm{a}-f_{0}$ & $\tilde{g} g$ & glueball $0^{++}$ \\
\hline 1.75 & $950(87)$ & $1070(123)$ & $1091(62)$ & $1319(120)$
\end{tabular}

Table 1: Bound state masses in units of $\mathrm{MeV}$, extrapolated to vanishing gluino mass.

The values of the extrapolated masses in QCD units are given in Table 1. In contrast to previous results, which were afflicted by larger systematic errors, our recent results are consistent with the emergence of a mass-degenerate chiral supermultiplet.

This is an important indication that this supersymmetric theory can be simulated on the lattice and nontrivial non-perturbative results are consistent with the theoretical prediction of an absent spontaneous supersymmetry breaking [1]. 


\section{Acknowledgements}

This project is supported by the German Science Foundation (DFG) under contract Mu 757/16. The authors gratefully acknowledge the computing time granted by the John von Neumann Institute for Computing (NIC) and provided on the supercomputers JUQUEEN and JUROPA at Jülich Supercomputing Centre (JSC). Further computing time has been provided by the compute cluster PALMA of the University of Münster.

\section{References}

[1] E. Witten, Nucl. Phys. B 202 (1982) 253.

[2] D. Amati, K. Konishi, Y. Meurice, G. C. Rossi, G. Veneziano, Phys. Rept. 162 (1988) 169.

[3] K. Demmouche, F. Farchioni, A. Ferling, I. Montvay, G. Münster, E. E. Scholz, J. Wuilloud, Eur. Phys. J. C 69 (2010) 147 [arXiv: 1003.2073 [hep-lat]].

[4] G. Bergner, I. Montvay, G. Münster, U. D. Özugurel, D. Sandbrink, PoS(Lattice 2011) 055 [arXiv: 1111.3012 [hep-lat]].

[5] G. Bergner, T. Berheide, I. Montvay, G. Münster, U. D. Özugurel, D. Sandbrink, JHEP 1209 (2012) 108 [arXiv: 1206.2341 [hep-lat]].

[6] G. Bergner, I. Montvay, G. Münster, U. D. Özugurel, D. Sandbrink, PoS (Lattice 2012) 042 [arXiv: 1210.7767 [hep-lat]].

[7] G. Bergner, I. Montvay, G. Münster, U. D. Özugurel, D. Sandbrink, JHEP, in press [arXiv: 1304.2168 [hep-lat]].

[8] G. Bergner, JHEP 1001 (2010) 024 [arXiv: 0909.4791 [hep-lat]].

[9] S. Catterall, D. B. Kaplan, M. Ünsal, Phys. Rept. 484 (2009) 71 [arXiv: 0903.4881 [hep-lat]].

[10] G. Curci, G. Veneziano, Nucl. Phys. B 292 (1987) 555.

[11] K. G. Wilson, Phys. Rev. D 10 (1974) 2445.

[12] J. Giedt, R. Brower, S. Catterall, G. T. Fleming, P. Vranas, Phys. Rev. D 79 (2009) 025015 [arXiv: 0810.5746 [hep-lat]].

[13] M. G. Endres, Phys. Rev. D 79 (2009) 094503 [arXiv: 0902 . 4267 [hep-lat]].

[14] S. W. Kim et al. [JLQCD Collaboration], PoS (Lattice 2011) 069 [arXiv: 1111.2180 [hep-lat]].

[15] I. Montvay, E. Scholz, Phys. Lett. B 623 (2005) 73 [arXiv: hep-lat/ 0506006 ].

[16] R. Kirchner et al. [DESY-Münster Collaboration], Phys. Lett. B 446 (1999) 209 [arXiv: hep-lat/9810062].

[17] I. Campos et al. [DESY-Münster Collaboration], Eur. Phys. J. C 11 (1999) 507 [arXiv: hep-lat/9903014].

[18] G. S. Bali, S. Collins, A. Schäfer, Comput. Phys. Commun. 181 (2010) 1570 [arXiv: 0910 . 3970 [hep-lat]].

[19] G. Veneziano, S. Yankielowicz, Phys. Lett. B 113 (1982) 231.

[20] G. R. Farrar, G. Gabadadze, M. Schwetz, Phys. Rev. D 58 (1998) 015009 [arXiv: hep-th/9711166].

[21] C. Morningstar, M. J. Peardon, Phys. Rev. D 69 (2004) 054501 [arXiv: hep-lat / 0311018 ]. 